\title{
Emergent versus Elective Cholecystectomy: Conversion Rates and Outcomes
}

\author{
Kathleen B. To,, Jill R. Cherry-Bukowiec,, Michael J. Englesbe, ${ }^{2}$ Michael N. Terjimanian, \\ Cai Shijie, ${ }^{2}$ Darrell A. Campbell, Jr., ${ }^{2}$ and Lena M. Napolitano ${ }^{1}$
}

\begin{abstract}
Background: Laparoscopic cholecystectomy (LC) is the procedure of choice for treatment of cholelithiasis/ cholecystitis. Conversion rates (CR) to open cholecystectomy (OC) have been reported previously as $5-15 \%$ in elective cases, and up to $25 \%$ in patients with acute cholecystitis. We examined the CR in a tertiary-care academic hospital and a statewide surgery quality collaborative, and to compare complications and outcomes in elective and emergency cholecystectomy.

Methods: Prospective data were obtained from: 1) Non-Trauma Emergency Surgery (NTE) database of all emergent cholecystectomies 1/1/2008-12/31/2009; and 2) Michigan Surgical Quality Collaborative (MSQC) database with a random sample of 20-30\% of all operations performed 1/1/2005-12/31/2010, including both University of Michigan (UM) data and statewide data from 34 participating hospitals. Patient characteristics, $C R$, and outcomes were compared for emergent vs. elective cases.

Results: Non-trauma ES patients had a mean hospital length of stay (HLOS) of $4.9 \mathrm{~d}$. Open cholecystectomyHLOS was greater (4.0, LC; 7.9 laparoscopic converted to open cholecystectomy; 8.7, OC, p<0.0001); mortality was $0.35 \%$ and CR was $17.5 \%$. In the UM-MSQC dataset, OC-HLOS was greater (6.8 OC vs. 4.6 LC, p<0.001); mortality was $0.65 \%$; CR was $9.1 \%$ in elective cases and $14.9 \%$ in emergent cases. CR was almost two-fold higher [17.5\% of all NTE cholecystectomies vs. 9.1\% of UM-MSQC elective cholecystectomies $(\mathrm{p}=0.00078)]$. The statewide MSQC cholecystectomy data showed significantly increased HLOS in emergent cholecystectomy patients ( 4.34 vs. 2.65 d; p < 0.0001$)$. Morbidity ( 8.8 vs. $3.7 \%)$ and mortality $(2.6$ vs. $0.5 \%)$ rates were also significantly higher in emergent vs. elective cholecystectomies $(\mathrm{p}<0.0001)$.

Conclusion: In NTE patients requiring cholecystectomy, CR is almost two-fold higher but is lower than in reports published previously (25\%). However, there is a wide variability in mortality and morbidity for emergency cholecystectomy in both unadjusted and risk-adjusted analyses. Further studies are required to determine modifiable risk factors to improve outcomes in emergency cholecystectomy.
\end{abstract}

W ITH ADVANCES IN LAPAROSCOPY OVER THE PAST two decades, laparoscopic cholecystectomy (LC) has become the procedure of choice for the treatment of most gallbladder pathology. In the elective outpatient setting, LC is often a same-day procedure. When conversion to open surgery is required, however, there are associated increases in length of stay (LOS), morbidity, and mortality. The national conversion rate $(\mathrm{CR})$ to open cholecystectomy is reported between $5-15 \%$ of all attempted LCs [1-5]. In the subset of patients who present with acute cholecystitis, the CR approaches $25 \%$ in previous reports [5]
Although acute gallbladder pathology (e.g. acute cholecystitis, gallstone pancreatitis) and open cholecystectomy (OC) are associated with adverse post-operative outcomes [6], recent data are sparse regarding $C R$ and outcomes in patients requiring emergency surgery. The objective of this study was to examine CRs and outcomes in patients requiring emergent vs. elective cholecystectomy. We hypothesized that patients who required emergent cholecystectomy would have a higher laparoscopic-to-open cholecystectomy CR, associated with increased hospital LOS, morbidity, and mortality, compared with elective cholecystectomy patients.

${ }^{1}$ Division of Acute Care Surgery, ${ }^{2}$ Division of Transplant Surgery, University of Michigan Department of Surgery, Ann Arbor, Michigan. Presented at the Thirty-second Annual Meeting of the Surgical Infection Society, Dallas, Texas, April 18-21, 2012. 


\section{Patients and Methods}

Data for this study were obtained from two sources: 1) the Non-Trauma Emergency (NTE) database at the University of Michigan; and 2) the Michigan Surgical Quality Collaborative (MSQC) database for the state of Michigan.

\section{Non-Trauma Emergency (NTE) database}

In July 2005, a NTE surgery service was created at the University of Michigan to be primarily responsible for evaluation and disposition of all urgent and emergent inpatient general surgery consults and emergency department general surgical consults, with admission to the NTE surgery service when appropriate. Data for the NTE database were collected prospectively by a dedicated trauma registrar and intensive care unit (ICU) coordinator daily. Overall case mix and patient characteristics have been reported previously [7]. From this database, we identified patients who had undergone LC or OC between January 1, 2008, and December 31, 2009, and reviewed retrospectively this subset of patients. Patient demographics (age, gender, and race), hospital LOS, and mortality data were extracted from the database. Morbidity data were not available in this database. Patient medical registration numbers were used as a cross-reference to obtain operating room (OR) times from the OR systems database; times reported reflect the total patient room time in the OR. Operative reports were reviewed manually by the authors to verify the procedure performed, and also to screen for conversion to an open procedure and reason for the conversion.

\section{Michigan Surgical Quality Collaborative database}

The Michigan Surgical Quality Collaborative database (MSQC) is a statewide surgical quality collaborative based on the American College of Surgeons (ACS) National Surgical Quality Improvement Program (NSQIP) that was created as a partnership among the ACS, 34 participating Michigan hospitals, and Blue Cross and Blue Shield of Michigan/The Blue Care Network, which funds the effort fully, with the goals of establishing an infrastructure for data collection, collaboration, and ad hoc quality improvement efforts within the state [8]. This collaborative is one of several to have demonstrated the effectiveness of regional collaboratives in improving the quality of surgical care [9]. Within the MSQC, there has been an absolute morbidity reduction in elective general and vascular surgery operations of $9.0 \%$ [10]. The MSQC has now initiated a focus on quality improvement in emergency general surgery in the state of Michigan.

The MSQC data are collected prospectively by a dedicated Surgical Clinical Nurse Reviewer who reviews operative and anesthesiology notes to stratify cases as emergent or elective, as defined by the ACS-NSQIP. Urgent cases are included in the elective cohort. Approximately $20-30 \%$ of all cases performed are entered into the MSQC database, with a robust range of clinical patient data points. Data collected include: Patient demographics, operation performed as defined by Current Procedural Terminology (CPT) codes (Table 1), operative times, preoperative and postoperative laboratory values, comorbid conditions, discharge/disposition information, American Society of Anesthesiologists classification, wound classification, mortality, and morbidity (morbidity parameters listed in Table 2) [11,12]. From January 1, 2005, to
Table 1. Current Procedural Terminology (CPT) Codes Used

Laparoscopic Cholecystectomy

47562 Laparoscopy, surgical; cholecystectomy

47563 Laparoscopy, surgical; cholecystectomy with cholangiography

47564 Laparoscopy, surgical; cholecystectomy with exploration of common bile duct

Open Cholecystectomy

47600 Cholecystectomy

47605 Cholecystectomy; with cholangiography

47610 Cholecystectomy with exploration of common bile duct

47612 Cholecystectomy with exploration of common bile duct; with choledochoenterostomy

47620 Cholecystectomy with exploration of common bile duct; with transduodenal sphincterotomy or sphincteroplasty, with or without cholangiography

December 31, 2010, the MSQC database was queried to extract cholecystectomy data by CPT codes. Cases were identified a priori as emergent or elective at time of data entry. The hospital LOS, 30-d post-operative morbidity, and mortality were assessed. Morbidity was defined as at least one case of the following: acute kidney injury, bleeding leading to transfusion, cardiac arrest, neurologic event (cerebrovascular accident [CVA], coma lasting more than $24 \mathrm{~h}$, or peripheral

Table 2. Post-Operative Complication List (Morbidity) For the Michigan Surgical Quality Collaborative (MSQC) Database

\begin{tabular}{ll}
\hline 1 & Acute kidney injury \\
2 & Bleeding/transfusions \\
3 & Cardiac arrest requiring CPR \\
4 & Coma $>24$ h \\
5 & Deep incisional SSI \\
6 & DVT/thrombophlebitis \\
8 & Graft/prosthesis/flap failure \\
12 & Myocardial infarction \\
13 & On ventilator $>48$ h \\
14 & Other cardiac occurrence \\
15 & Other CNS occurrence \\
16 & Other occurrence \\
17 & Other respiratory occurrence \\
18 & Other urinary tract occurrence \\
19 & Peripheral nerve injury \\
20 & Pneumonia \\
22 & Pulmonary embolism \\
23 & Progressive renal insufficiency \\
26 & Stroke/CVA \\
27 & Superficial incisional SSI \\
28 & Sepsis \\
29 & Unplanned intubation \\
30 & Urinary tract infection \\
31 & Wound disruption \\
32 & Organ/space SSI \\
33 & Other wound occurrence \\
34 & Septic shock \\
35 & SIRS \\
\hline
\end{tabular}

$\mathrm{CNS}=$ central nervous system; $\mathrm{CPR}=$ cardiopulmonary resuscitation; CVA = cerebro-vascular accident; SIRS = systematic inflammatory response syndrome; SSI = surgical site infection. 
Table 3. Non-Trauma Emergency (NTE) Service Cholecystectomy Data for the University of Michigan Medical Center

Non Trauma Emergency Patients Undergoing Cholecystectomy (Jan 1, 2008 to Dec 31, 2009)

\begin{tabular}{lcccc}
\hline & $\begin{array}{c}\text { Overall NTE } \\
\text { cholecystectomy } \\
n=279\end{array}$ & $\begin{array}{c}\text { LC } \\
n=217(77.78 \%)\end{array}$ & $\begin{array}{c}\text { CONV } \\
n=46(16.49 \%)^{\ddagger}\end{array}$ & $n=16(5.73 \%)$ \\
\hline $\begin{array}{l}\text { Gender (\%) } \\
\text { Male }\end{array}$ & $96(34.4 \%)$ & $63(29 \%)$ & $26(56.5 \%)$ & $7(43.8 \%)$ \\
Female & $183(65.6 \%)$ & $154(71 \%)$ & $20(43.5 \%)$ & $9(56.2 \%)$ \\
Age, yrs & $45.4(18-91)$ & $43.8(18-91)$ & $51.1(19-85)$ & $50.2(26-74)$ \\
$\begin{array}{l}\text { Average (range) } \\
\text { Operative Time, min (in-room time }\end{array}$ & & & & \\
$\quad$ to post-anesthesia care unit) & $169.0(75-439)$ & $156.7^{\S}(74-439)$ & $216.7^{\S}(131-411)$ & $199.1^{\S}(94-424)$ \\
$\begin{array}{l}\text { Average (Range) } \\
\text { Hospital Length of Stay (HLOS), d }\end{array}$ & 4.9 & $4.0^{*}$ & 0 & $8.7^{*}$ \\
Mortality (\%) & $1(0.35 \%)$ & 0 & $1(6.25 \%)$ \\
\hline
\end{tabular}

${ }^{¥} \mathrm{CONV}$ was $16.5 \%$ of all NTE cholecystectomies; conversion rate was calculated as CONV/(LC+CONV)=17.5\% of all laparoscopic attempts.

${ }^{\S}$ Operative times were significantly less in laparoscopic cholecystectomy: LC vs. OC ( $\left.p<0.0001\right)$; LC vs. CONV ( $\left.p=0.0036\right)$.

*HLOS significantly greater in open cholecystectomies: LC vs. OC ( $p<0.001)$; LC vs. CONV ( $<<0.0001)$.

$\mathrm{CONV}=$ laparoscopic surgery cases converted to open cholecystectomy; HLOS=hospital length of stay; LC=laparoscopic cholecystectomy; NTE = non-trauma emergency; $\mathrm{OC}=$ open cholecystectomy.

nerve injury), surgical site infection (superficial, deep, or organ space), deep venous thrombosis, prosthetic graft failure, myocardial infarction, renal insufficiency, pulmonary embolism, stroke, sepsis, septic shock, unplanned intubation, wound disruption, and ventilator dependence for more than $48 \mathrm{~h}$. Urinary tract infection was excluded as a morbidity outcome for the analysis.

\section{Statistical Analysis}

Descriptive statistics were computed for the study cohort. Continuous variables were summarized by the mean and standard deviation, whereas categorical variables were summarized with frequency tables. Continuous variables were compared using Student's t-test, while chi square and Fisher exact tests were used to compare categorical and dichotomous variables, respectively, with a $\mathrm{p}$ value of 0.05 considered significant. Relationships between the proportion of emergent surgery and morbidity or mortality were assessed using linear regression techniques. Logistic regression analysis was used to identify factors associated independently with morbidity and mortality to provide risk-adjusted morbidity and mortality outcome data. A morbid outcome was defined as the incidence of any of the outcomes previously listed. The 42 covariates available in the MSQC included: Age, alcoholism, angina, ascites, race, bleeding diathesis, body mass index, cancer, chemotherapy, congestive heart failure, central nervous system deficit, chronic obstructive pulmonary disease, cerebrovascular accident (CVA) resulting in residual neurological deficit, CVA resulting in no neurological deficit, diabetes mellitus, dialysis, dyspnea, esophageal varices, gangrene, gender, hemiplegia, hypertension, impaired sensorium, open wound, paraparesis, percutaneous transluminal coronary angioplasty, pre-operative myocardial infarction,

Table 4. Cholecystectomy in the Michigan State Quality Collaborative (MSQC) Database, Stratified by Elective vs. Emergent. Urgent Cases Were Grouped With Elective Cases

\begin{tabular}{|c|c|c|c|c|}
\hline & $\begin{array}{c}\text { Statewide } \\
\text { MSQC Elective }\end{array}$ & $\begin{array}{c}\text { Statewide MSQC } \\
\text { Emergent }\end{array}$ & $\begin{array}{l}\text { Univ. Michigan } \\
\text { MSQC Elective }\end{array}$ & $\begin{array}{l}\text { Univ. Michigan } \\
\text { MSQC Emergent }\end{array}$ \\
\hline $\mathrm{N}$ & 16,697 & 1,409 & 672 & 100 \\
\hline Mean age, yrs & 49.8 & 51.6 & 47.8 & 45.9 \\
\hline Male & $27.5 \%(4,599)$ & $40.2 \%(567)$ & $32.5 \%(219)$ & $34.0 \%(34)$ \\
\hline Operative time, $\min$ & 96.6 & 112.6 & 145.9 & 156.2 \\
\hline LC & $90.9 \%(15,185)$ & $76.7 \%(1,080)$ & $82.8 \%(557)$ & $80.0 \%(80)$ \\
\hline $\begin{array}{l}\text { Conversion rate (converted/laparoscopic } \\
\text { attempts) }\end{array}$ & NA & NA & $9.1 \% ¥$ & $14.9 \%$ \\
\hline HLOS, d & $2.65^{\S}$ & $4.34^{\S}$ & 3.73 & 5.44 \\
\hline Morbidity (\%) & $3.7 \% \S$ & $8.8 \% \S$ & $4.9 \%$ & $10.0 \%$ \\
\hline Mortality (\%) & $0.5 \% \S$ & $2.6 \% \S$ & $0.6 \%$ & $1.0 \%$ \\
\hline
\end{tabular}

${ }^{¥}$ Conversion rate was calculated as converted cases $(\mathrm{CONV}) /(\mathrm{LC}+\mathrm{CONV}) \cdot \mathrm{p}=0.0 .053$ for conversion rate for elective vs. emergent. ${ }^{\S} \mathrm{p}<0.0001$ for HLOS, morbidity and mortality in statewide MSQC elective vs. emergent cholecystectomy patients.

$\mathrm{CONV}=$ laparoscopic surgery cases converted to open cholecystectomy; HLOS=hospital length of stay; LC=laparoscopic cholecystectomy; MSQC = michigan state quality collaborative. 
preoperative sepsis, pre-operative functional status, previous cardiac surgery, peripheral vascular disease, quadriparesis, radiotherapy, renal insufficiency, smoking, steroid use, transient ischemic attack, total operation time, transfer from home, transfusion, unintended weight loss, and ventilator dependence. All candidate predictors were entered into the model, and stepwise backwards selection was used to select a subset of adjustment covariates. The c-statistic was used to gauge the model's predictive accuracy.

The output from the logistic regression analysis was used to derive the adjusted morbidity and mortality ratios. These were calculated by dividing the predicted number of morbidity or mortality cases (sum of predicted frequencies per site) by the total number of observed cases per site. The $95 \%$ confidence intervals for these rates were calculated in an identical fashion, by dividing the upper and lower estimates by total cases per site. A two-sided significance level of $\alpha=0.001$ was used in all analyses. All statistical computations were performed in SAS v9.2 (SAS Institute; Cary, NC).

\section{Results}

\section{Non-trauma Emergency Surgery Data}

Between January 2008 and December 2009, there were 1708 NTE admissions, and 1202 patients required surgical intervention. Cholecystectomy was performed in 279 (23\%) patients: 217 (77.8\%) were laparoscopic (LC), 16 (5.7\%) were open (OC), and 46 (16.5\% of all NTE patients, $17.5 \%$ of all laparoscopic attempts) were laparoscopic converted to open cholecystectomy (CONV; Table 3). Overall, female gender was more common ( $34.4 \%$ male, $65.6 \%$ female). Mean age was 45.4 y (range 18-91). Operative times were significantly less in laparoscopic cases; however, the range in OR times was similar. HLOS was significantly greater in patients undergoing open cholecystectomy (mean HLOS 4.9 days overall, 4.0 LC, 7.9 CONV, 8.7 OC; $p<0.0001 \mathrm{LC}$ vs. OC and $\mathrm{p}=0.0036$ in LC vs. CONV). Overall hospital mortality was $0.35 \%$; the incidence of death was too small to detect any significant differences identified amongst the three cohorts.

\section{Michigan Surgical Quality Collaborative Data}

Statewide MSQC Data. From January 2005 to December 2010, 190,826 operations were entered into the MSQC data-

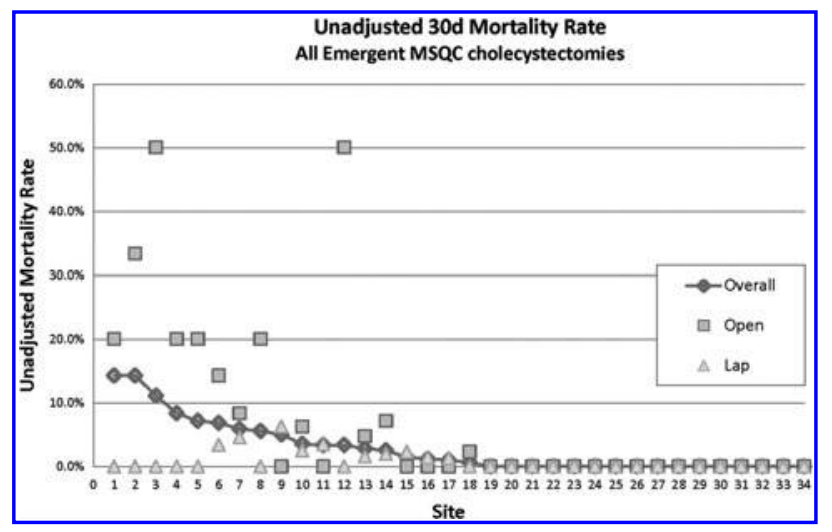

FIG. 1. Unadjusted 30-d mortality rate among all emergent Michigan Surgical Quality Collaborative database cholecystectomies performed between 2006-2010.

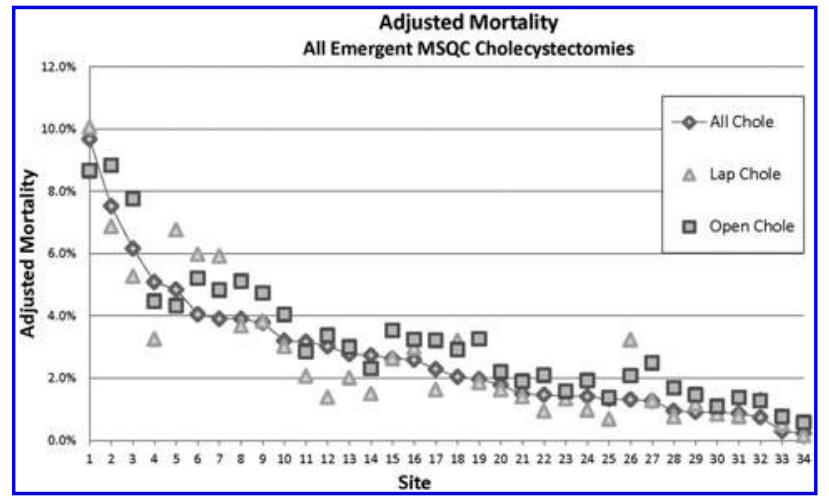

FIG. 2. Risk-adjusted 30-d mortality rate among all emergent Michigan Surgical Quality Collaborative database cholecystectomies performed between 2006-2010. Adjustment covariates: pre-operative sepsis, age, pre-operative functional status, pre-operative ventilator, pre-operative ascites.

base. Cholecystectomies accounted for 18,106 cases $(9.52 \%$ of total cases; Table 4), with 16,697 (92.22\%) elective and 1,409 (7.78\%) emergent cases. Laparoscopic cholecystectomy was performed in $90.9 \%$ of elective cases vs. $78.7 \%$ of emergent cases. Hospital LOS was significantly increased in emergent cholecystectomy patients ( 4.34 vs. $2.65 \mathrm{~d}$ ). Morbidity (8.8 vs. $3.7 \%$ ) and mortality ( 2.6 vs. $0.5 \%$ ) rates were also significantly higher in emergent vs. elective cases $(\mathrm{p}<0.0001)$.

Unadjusted and risk-adjusted mortality (Figs. 1 and 2) and morbidity (Figs. 3 and 4) data were examined for the statewide MSQC data for all emergent cholecystectomy patients. In the unadjusted analysis, OC was associated with higher mortality and morbidity rates, but significant site-dependent variability was noted among the 34 hospitals.

In the risk-adjusted analysis of emergent (Fig. 2 and Fig. 4) and elective cholecystectomy patients (Fig. 5 and Fig. 6), independent variables that impacted outcomes were adjusted to assess further the differences in mortality and morbidity. For mortality risk adjustment, adjustment covariates included pre-operative sepsis, age, pre-operative functional status, pre-operative ventilator, and pre-operative ascites. For morbidity risk adjustment, adjustment covariates included laparoscopic approach, total OR time, pre-

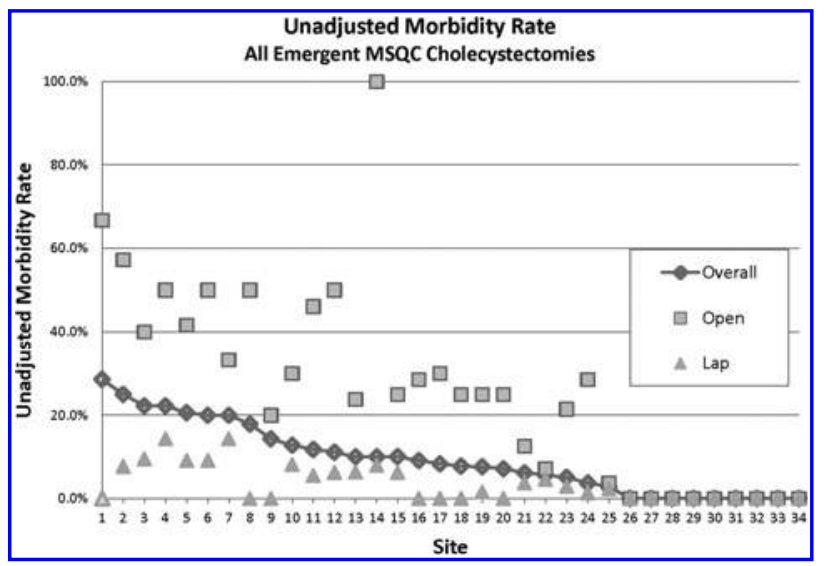

FIG. 3. Unadjusted 30-d morbidity rate among all emergent Michigan Surgical Quality Collaborative database cholecystectomies performed between 2006-2010. 


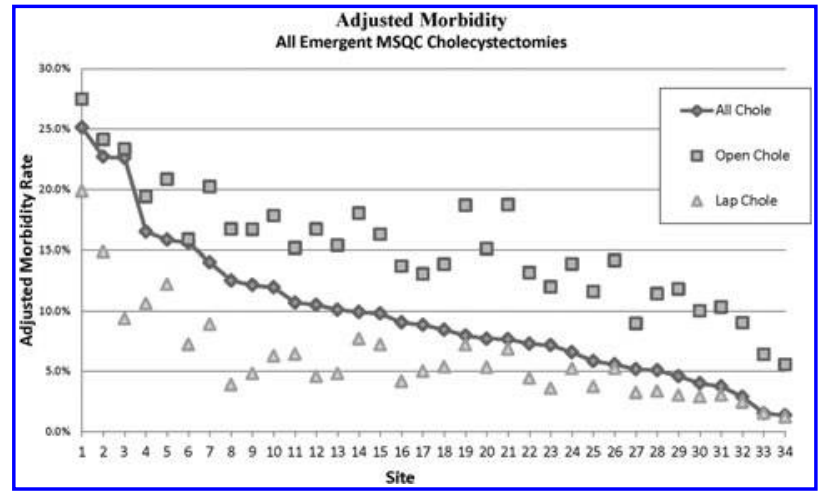

FIG. 4. Risk-adjusted 30-d morbidity rate among all emergent Michigan Surgical Quality Collaborative database cholecystectomies performed between 2006-2010. Adjustment covariates: Laparoscopic approach, total operating room time, pre-operative sepsis, age, pre-operative functional status, pre-operative pneumonia, pre-operative ascites, pre-operative sensorium, pre-operative cerebral vascular accident resulting in neurologic deficit.

operative sepsis, age, pre-operative functional status, preoperative pneumonia, pre-operative ascites, pre-operative sensorium, and pre-operative CVA resulting in neurologic deficit. These risk-adjusted data validate the unadjusted data and confirm significant site-dependent mortality rates (ranging from 0.1 to $10.0 \%$ ) and morbidity rates (ranging from 0.1 to $25 \%$ ). Furthermore, there is an overall increase in mortality and morbidity in patients who had emergency status or underwent OC.

University of Michigan MSQC Data. The University of Michigan MSQC data included 10,960 operations, of which $773(7.05 \%)$ were cholecystectomies. One case was dropped due to CPT/charting discrepancy. Of the remaining 772 cases, 672 were classified as elective and 100 as emergent cases (Table 4 and Table 5). Cholecystectomy patients were $67.4 \%$ female, with a mean age of $47.5 \mathrm{y}$ (range 18-94). Laparoscopic cholecystectomy was performed in $82.8 \%$ of elective cases vs. $80 \%$ of emergent cases, similar to the statewide MSQC data.

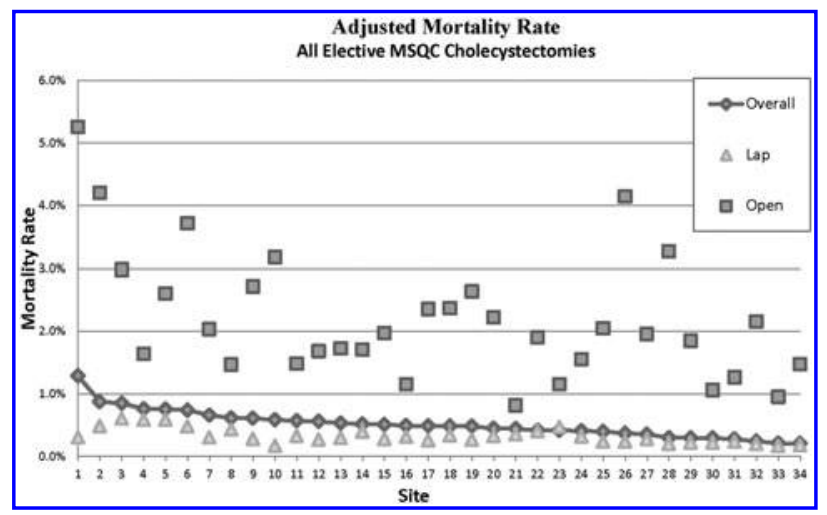

FIG. 5. Risk-adjusted 30-d mortality rate among all elective Michigan Surgical Quality Collaborative database cholecystectomies performed between 2006-2010. Adjustment covariates: Pre-operative sepsis, age, pre-operative functional status, pre-operative ventilator, pre-operative ascites.

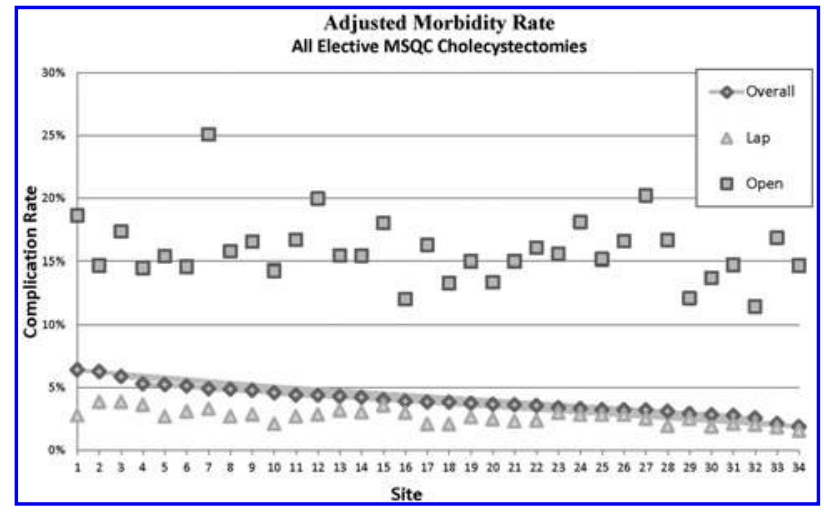

FIG. 6. Risk-adjusted 30-d morbidity rate among all elective Michigan Surgical Quality Collaborative database cholecystectomies performed between 2006-2010. Adjustment covariates: Laparoscopic approach, total operating room time, pre-operative sepsis, age, pre-operative functional status, pre-operative pneumonia, pre-operative ascites preoperative sensorium, pre-operative cerebral vascular accident resulting in neurologic deficit.

Hospital LOS (5.44 vs. 3.73 d), morbidity (10.0 vs. $4.9 \%$ ), and mortality (1.0 vs. $0.6 \%$ ) rates were all higher in emergent cases.

We next compared LC vs. OC outcomes (Table 5). Hospital LOS was greater for the OC group (6.8 OC vs $4.6 \mathrm{LC}$, $\mathrm{p}=0.006)$. Overall hospital mortality was $0.65 \%(0.6 \%$ elective, $1 \%$ emergent). Conversion rate from LC to OC was higher in the emergent cases (14.9\% in emergent cases vs. $9.1 \%$ in elective cases; $\mathrm{p}=0.053)$. Operative time was also higher in the emergent group vs. the elective group $(p=0.055)$ and in OC vs. LC ( $<<0.0001)$. Post-operative complications occurred in $56(7.3 \%)$ of 772 elective cholecystectomy patients, with superficial incisional (SSI) being the most common post-operative complication reported (Table 6).

\section{Comparison of NTE and University of Michigan MSQC data}

Conversion rates (LC to OC) were similar for NTE emergency patients (17.5\%) and University of Michigan MSQC emergency patients (14.9\%), and significantly higher than the CR in elective patients $(9.1 \% ; \mathrm{p}=0.00078$; Table 7$)$.

Operative duration was also significantly increased for NTE vs. MSQC elective cholecystectomy cases $(p<0.0001)$. No differences in age or gender were identified between these groups.

\section{Discussion}

Cholecystectomy is one of the most common operations performed on emergency surgical services. We described previously that cholecystectomy comprised $24 \%$ of all surgical cases on the NTE service at the University of Michigan. In this current study, we examined CR (LC to OC) and outcome in elective vs. emergent cholecystectomy patients at our institution and in the state of Michigan. Evaluation of outcomes in emergency surgery is an important initiative, because it provides the foundation of data for performance improvement in this group of patients. Previous performance improvement efforts have focused only on elective general 
Table 5. Michigan Surgical Quality Collaborative (MSQC) Cholecystectomy Data For the University of Michigan Medical Center

University of Michigan MSQC patients undergoing elective and emergency cholecystectomy (Jan 1, 2005 to Dec 31, 2010)

\begin{tabular}{|c|c|c|c|c|}
\hline & \multicolumn{2}{|c|}{ Elective cholecystectomy } & \multicolumn{2}{|c|}{ Emergency cholecystectomy } \\
\hline & \multicolumn{2}{|c|}{$n=672$} & \multicolumn{2}{|c|}{$n=100$} \\
\hline & $\begin{array}{c}L C \\
n=557\end{array}$ & $\begin{array}{c}\text { OC } \\
n=115\end{array}$ & $\begin{array}{c}L C \\
n=80\end{array}$ & $\begin{array}{c}\text { OC } \\
n=20\end{array}$ \\
\hline \multicolumn{5}{|l|}{ Gender (\%) } \\
\hline Male & 155 (27.8\%) & $63(54.8 \%)$ & $24(30 \%)$ & $10(50 \%)$ \\
\hline Female & $402(72.2 \%)$ & $52(45.2 \%)$ & $56(70 \%)$ & $10(50 \%)$ \\
\hline $\begin{array}{l}\text { Age (yrs) } \\
\text { average (range) }\end{array}$ & $45.9(18-92)$ & $57.0(19-85)$ & $42.1(18-93)$ & $60(26-94)$ \\
\hline \multicolumn{5}{|l|}{$\begin{array}{l}\text { Operative time (min, surgical } \\
\text { start to finish) }\end{array}$} \\
\hline average (range) & $96.7(28-288)^{\S}$ & $155.9(52-364)^{\S}$ & $109.6(51-232)^{\S}$ & $140.8(55-220)^{\S}$ \\
\hline Hospital length of stay (d) & 5.9 & 6.2 & 4.6 & 6.2 \\
\hline Morbidity (\%) & $2.5 \%$ & $2.4 \%$ & $5 \%$ & $5 \%$ \\
\hline Mortality (\%) & $0.3 \%$ & $0.3 \%$ & $1 \%$ & 0 \\
\hline
\end{tabular}

surgery, but recent data confirm that a far greater proportion of morbidity and mortality occurs in emergency general surgery patients, providing support for more in-depth analyses of our emergency general surgery cases [5,13].

In this study, we reviewed cholecystectomy data from two different perspectives and two different databases available at the University of Michigan. The NTE database captured all patients admitted or seen in consultation for acute gallbladder pathology (most commonly acute cholecystitis or gallstone pancreatitis) who underwent cholecystectomy subsequently. By comparison, we examined University of Michigan institutional data from the MSQC database, which includes a random sampling that captures both outpatient elective cholecystectomies for symptomatic cholelithiasis, as well as patients undergoing emergency cholecystectomy. These data

Table 6. Michigan Surgical Quality Collaborative (MSQC) CHOLECYSTECTOMY DATA FOR THE UNIVERSITY of Michigan Medical Center - Morbidity Outcomes $(\mathrm{N}=772)$

\begin{tabular}{lc} 
Complication & \# patient \\
\hline Acute kidney injury & 2 \\
Bleeding/transfusions & 2 \\
Cardiac arrest requiring CPR & 1 \\
DVT/thrombophlebitis & 4 \\
On ventilator $>48$ h & 5 \\
Pneumonia & 4 \\
Progressive renal insufficiency & 3 \\
Superficial incisional SSI & 16 \\
Sepsis & 7 \\
Unplanned intubation & 4 \\
Wound disruption & 1 \\
Organ/space SSI & 4 \\
Septic shock & 3 \\
\hline
\end{tabular}

$\mathrm{CPR}=$ cardiopulmonary resuscitation; $\mathrm{DVT}=$ deep venous thrombosis; SSI = surgical site infection. confirmed that CRs for the emergent cases were higher than the elective cases but surprisingly there was no significant difference in morbidity or mortality in cases performed at the University of Michigan.

The University of Michigan institutional data also confirmed lower morbidity and mortality rates than previously reported. In their review of the ACS-NSQIP data for emergency cholecystectomies performed from 2005-2008, Ingraham et al. reported overall morbidity and mortality rates of $8.64 \%$ and $1.80 \%$ respectively [5]. By comparison, our overall morbidity and mortality rates for the University of Michigan MSQC database were 5\% and 1\% for emergent cholecystectomies, with even lower rates in the elective cholecystectomy group. Morbidity data were not available for the NTE database, but mortality was $0.35 \%$ in this specific emergency cholecystectomy cohort.

In contrast to our University of Michigan institutional data (NTE and MSQC data), the statewide MSQC data confirmed that morbidity ( 8.8 vs. $3.7 \%$ ) and mortality (2.6 vs. $0.5 \%$ ) rates were significantly higher in emergent vs. elective cases. Risk-adjusted mortality/morbidity data also confirmed wide variability and significant differences in sitedependent mortality rates (ranging from 0.1 to $10.0 \%$ ) and morbidity rates (ranging from 0.1 to $25 \%$ ) in all emergency cholecystectomy cases.

There are several limitations to our study. All data for both the NTE and the MSQC database were collected prospectively, but analyzed retrospectively. The MSQC database did not include all cholecystectomy cases, either at the University of Michigan or statewide, thus selection bias is a possible confounding variable. In the case of the NTE database, operations were identified via the electronic medical record. It is possible that not all cholecystectomies performed were captured and the total number of cases may be under-represented.

Additionally, although the service is designed to care for urgent and emergent general surgical patients, on occasion 
Table 7. Non-Trauma Emergency (NTE) Service and Michigan Surgical Quality Collaborative (MSQC) Cholecystectomy Data for the University of Michigan Medical Center, Conversion Rates

Rates of Conversion from Laparoscopic to Open Cholecystectomy

\begin{tabular}{lccc}
\hline & & \multicolumn{2}{c}{ Univ. Michigan MSQC database 2005-2010 } \\
\cline { 3 - 4 } & NTE 2008-2009 & Elective Cases & Emergent Cases \\
\hline $\begin{array}{l}\text { Converted cases / Laparoscopic attempts } \\
\text { (conversion rate) }\end{array}$ & $\mathrm{n}=46 / 263^{¥}$ & $\mathrm{n}=56 / 613^{¥}$ & $\mathrm{n}=14 / 94^{\ddagger}$ \\
Gender & $(17.5 \%)$ & $(9.1 \%)$ & $(14.9 \%)$ \\
$\quad$ & & $7(50 \%)$ \\
$\quad$ Male & $26(56.5 \%)$ & $33(58.9 \%)$ & $7(50 \%)$ \\
Memale & $20(43.5 \%)$ & $23(41.1 \%)$ & 57.1 \\
Mean Age (yrs) & 51.1 & 57.1 & $147.0^{\S}$ \\
Time (min) & $216.7^{\S}$ & $164.8^{\S}$ & \\
\hline
\end{tabular}

${ }^{¥}$ Conversion rate was calculated as a ratio of converted cases to all laparoscopic attempts: CONV/(LC+CONV). Conversion rates were statistically significant for emergent vs. elective cases: 1 ) NTE vs. elective MSQC cases ( $p=0.00078) ; 2)$ MSQC elective vs. MSQC emergent cases $(p=0.0941) ; 3)$ no statistically significant difference for NTE vs. MSQC emergent cases $(p=0.63)$.

§operative times were defined as total patient time in room for the NTE database; for the MSQC database operative times were defined as surgical incision to close: 1) NTE vs. elective MSQC cases ( $p<0.0001) ; 2)$ MSQC elective vs. MSQC emergent cases ( $p=0.055) ; 3)$ NTE vs. MSQC emergent cases $(\mathrm{p}=0.036)$.

NTE = Non-Trauma Emergency Surgery database; MSQC = Michigan Surgical Quality Collaborative; CONV=laparoscopic surgery cases converted to open cholecystectomy; LC=laparoscopic cholecystectomy.

outpatient cases may be scheduled into the NTE service time. There is no a priori definition in the NTE database for what constituted elective, urgent, or emergent surgical intervention. Additionally, one-half of the NTE faculty also cover the Trauma, Burn, and Emergency Surgery (TBE) service, and it is possible that some cases performed by the TBE service and therefore not captured properly into the NTE database. Operative times in the NTE database were extracted from the operating room system records retrospectively, and reflect total room time rather than actual surgery start-to-end time. Thus, the operating room times may be an over-estimate of the actual length of surgery. The University of Michigan MSQC data is a much broader sampling of all cases performed at the University Hospital, both on the elective general surgery services as well as the NTE and TBE services. The method for data entry is to capture all cases during a portion of each month, with a cap for the types of cases captured per month. The premise is to establish a random sampling of the total case load, but theoretically could result in sampling error. Some of the patient cases included in the University of Michigan MSQC database also overlap with the NTE database, but whereas the NTE data include all cholecystectomies from the NTE service, the University of Michigan MSQC database only has a partial inclusion of the NTE emergent cases.

In conclusion, although patients requiring emergent cholecystectomy have an almost two- fold higher risk CR (17.5\% NTE vs. 9.1\% elective MSQC), this is lower than in previous published reports $(25 \%)$ and supports the initial attempt at laparoscopy even in the emergent setting. Further, whereas emergency cholecystectomy is associated with longer operative times and hospital LOS, there was no difference in mortality identified in our NTE and MSQC institutional data. In contrast, statewide MSQC data confirm wide site-specific variability in risk-adjusted outcomes (30-d mortality and morbidity) for emergency cholecystectomy, and additional in-depth analyses are underway to determine the factors associated with high-outlier vs. low-outlier hospitals. Data from these analyses will be able to be used to initiate performance improvement efforts in emergency cholecystectomy.

\section{Author Disclosure Statement}

No competing financial interests exist.

\section{References}

1. Kaafarani HM, Smith TS, Neumayer L, et al. Trends, outcomes, and predictors of open and conversion to open cholecystectomy in Veterans Health Administration hospitals. Am J Surg 2010;200:32-40.

2. Wiseman JT, Sharuk MN, Singla A, et al. Surgical management of acute cholecystitis at a tertiary care center in the modern era. Arch Surg 2010;145:439-444.

3. Sakpal SV, Bindra SS, Chamberlain RS. Laparoscopic cholecystectomy conversion rates two decades later. ISLS 2010;14:476-483.

4. Banz V, Gsponer T, Candinas D, Guller U. Population-based analysis of 4113 patients with acute cholecystitis: defining the optimal time-point for laparoscopic cholecystectomy. Ann Surg 2011;254:964-970.

5. Livingston EH, Rege RV. A nationwide study of conversion from laparoscopic to open cholecystectomy. Am J Surg 2004;188:205-211.

6. Ingraham $\mathrm{AM}$, Cohen ME, Raval MV, et al. Comparison of hospital performance in emergency versus elective general surgery operations at 198 hospitals. J Am Coll Surg 2011;212:20-28.

7. Cherry-Bukowiec JR, Miller BS, Doherty GM, et al. Nontrauma emergency surgery: optimal case mix for general surgery and acute care surgery training. J Trauma 2011;71: 1422-1426.

8. Campbell DA Jr, Kubus JJ, Henke PK, et al. The Michigan Surgical Quality Collaborative: A legacy of Shukri Khuri. Am J Surg 2009;198(5 Suppl):S49-S55. 
9. Share DA, Campbell DA, Birkmeyer N, et al. How a regional collaborative of hospitals and physicians in Michigan cut costs and improved the quality of care. Health Aff (Millwood) 2011;30:636-645.

10. Campbell DA, Jr., Englesbe MJ, Kubus JJ, et al. Accelerating the pace of surgical quality improvement:the power of hospital collaboration. Arch Surg 2010;145:985-91.

11. Englesbe MJ, Dimick JB, Sonnenday CJ, et al. The Michigan surgical quality collaborative: will a statewide quality improvement initiative pay for itself? Ann Surg 2007;246: 1100-1103.

12. Campbell DA, Jr., Englesbe MJ, Kubus JJ, et al. Accelerating the pace of surgical quality improvement: The power of hospital collaboration. Arch Surg 2010; 145:985-991.
13. Akinbami F, Askari R, Steinberg J, et al. Factors affecting morbidity in emergency general surgery. Am J Surg 2011; 201(4):456-462.

Address correspondence to: Dr. Kathleen B. To

Division of Acute Care Surgery Department of Surgery University of Michigan University Hospital 1C340 1500 East Medical Center Dr. Ann Arbor, MI 48109-0033

E-mail: KaTo@umich.edu 


\section{This article has been cited by:}

1. Elizabeth J. Lilley, John W. Scott, Wei Jiang, Anna Krasnova, Nikhila Raol, Navin Changoor, Ali Salim, Adil H. Haider, Joel S. Weissman, Eric B. Schneider, Zara Cooper. 2017. Intraoperative cholangiography during cholecystectomy among hospitalized medicare beneficiaries with non-neoplastic biliary disease. The American Journal of Surgery 214:4, 682-686. [Crossref]

2. Steven J. Coffin, Sean M. Wrenn, Peter W. Callas, Wasef Abu-Jaish. 2017. Three decades later: investigating the rate of and risks for conversion from laparoscopic to open cholecystectomy. Surgical Endoscopy 5. . [Crossref]

3. Donna Marie L. Alvino, Zhi Ven Fong, Colin J. McCarthy, George Velmahos, Keith D. Lillemoe, Peter R. Mueller, Peter J. Fagenholz. 2017. Long-Term Outcomes Following Percutaneous Cholecystostomy Tube Placement for Treatment of Acute Calculous Cholecystitis. Journal of Gastrointestinal Surgery 21:5, 761-769. [Crossref]

4. Roxanne L. Massoumi, Colleen M. Trevino, Travis P. Webb. 2017. Postoperative Complications of Laparoscopic Cholecystectomy for Acute Cholecystitis: A Comparison to the ACS-NSQIP Risk Calculator and the Tokyo Guidelines. World Journal of Surgery 41:4, 935-939. [Crossref]

5. Fabian Grass, Matthieu Cachemaille, Catherine Blanc, Nicolas Fournier, Nermin Halkic, Nicolas Demartines, Martin Hübner. 2016. Is standardized care feasible in the emergency setting? A case matched analysis of patients undergoing laparoscopic cholecystectomy. BMC Surgery 16:1. . [Crossref]

6. Kamila Rafaela Alves, Alessandra Carvalho Goulart, Roberto Marini Ladeira, Ilka Regina Souza de Oliveira, Isabela Martins Benseñor. 2016. Frequency of cholecystectomy and associated sociodemographic and clinical risk factors in the ELSA-Brasil study. Sao Paulo Medical Journal 134:3, 240-250. [Crossref]

7. Yi-Ren Hu, Jiang-Hua Pan, Xiao-Chun Tong, Ke-Qin Li, Sen-Rui Chen, Yi Huang. 2015. Efficacy and safety of Bmode ultrasound-guided percutaneous transhepatic gallbladder drainage combined with laparoscopic cholecystectomy for acute cholecystitis in elderly and high-risk patients. BMC Gastroenterology 15:1. . [Crossref]

8. Megan Sippey, John R. Pender, William H.H. Chapman, Mark L. Manwaring, Kevin R. Kasten, Walter E. Pofahl, Konstantinos Spaniolas. 2015. Delayed repair of obstructing ventral hernias is associated with higher mortality and morbidity. The American Journal of Surgery 210:5, 833-837. [Crossref]

9. Torben. Horn, Sara D. Christensen, Jakob. Kirkegård, Lars P. Larsen, Anders R. Knudsen, Frank V. Mortensen. 2015. Percutaneous cholecystostomy is an effective treatment option for acute calculous cholecystitis: a 10-year experience. $H P B$ 17:4, 326-331. [Crossref]

10. Andrew B. Peitzman, Gregory A. Watson, J. Wallis Marsh. 2015. Acute cholecystitis. Journal of Trauma and Acute Care Surgery 78:1, 1-12. [Crossref]

11. Erin Moran-Atkin, Miloslawa Stem, Anne O. Lidor. 2014. Surgery for diverticulitis is associated with high risk of in-hospital mortality and morbidity in older patients with end-stage renal disease. Surgery 156:2, 361-370. [Crossref] 